Our understanding of disorder is questioned with the discovery, on extending measurement ranges, that the acoustic properties of polycrystalline metals resemble those of glasses, for which significant deviations from predictions of the standard tunnelling model are also found.

\title{
Low-Temperature Acoustic Properties of Glasses and Polycrystals
}

Glasses exhibit low-temperature properties which are distinctively different to those of crystals [1]. For instance, the heat capacity below $1 \mathrm{~K}$ in the glassy state is substantially larger and has a different temperature dependence than in the crystalline state. The thermal conductivity in the glassy state is also much smaller and again shows a different temperature dependence. Other characteristic features of glasses are the temperature dependence of the velocity of sound $v$ and of the attenuation of sound waves (given by the internal friction $Q$ ), both of which have been studied extensively at temperatures $T$ above $20 \mathrm{mK}$ [2]. Finally, there is the similarity of properties of quite different glasses at $T<1 \mathrm{~K}$.

All the low-temperature properties of glasses have been successfully explained in terms of the phenomenological "two-level systems tunnelling model" [3]. It assumes that atoms or groups of atoms have more than one site in a disordered lattice, and that these sites are separated by energy barriers. At low temperatures, atoms or groups of atoms can tunnel through the potential wells separating their possible sites. In the simplest version of the model, only the tunnelling between the ground states of a double-well potential is considered. In glasses, owing to the random disorder, it is assumed that there is a wide distribution of tunnelling energies (leading to a constant density of tunnelling states) and a very wide distribution of relaxation times (experimentally observed to range from $10^{-9} \mathrm{~s}$ to $10^{6} \mathrm{~s}$ !).

\section{Several Issues}

Low-energy tunnelling excitations and their interactions with phonons and conduc-

Pablo Esquinazi joined the research staff of the Physikalisches Institut, Universität Bayreuth, D-95412, in 1988. He received his Ph.D. in 1983 from the Centro Atomico Bariloche in Argentina where he worked for a year after spending two years as a research assistant at the University of Heidelberg.

Reinhard König received his doctorate from the University of Bayreuth in 1993 where he remains on the research staff.

Frank Pobell has been a professor at the University of Bayreuth since 1983. He received his doctorate in 1965 from the TU Munich where he remained until becoming a professor at the University of Cologne and Director of the KFA Jülich in 1975. He has been an IUPAP Vice-President since 1990 and Editor of J. Low Temp. Physics since 1992. Professor Pobell won the 1988 Emil Warburg Prize and the 1992 Lise Meitner/Alex von Humboldt Prize.

\section{P. Esquinazi, R. König, F. Pobell \\ Physikalisches Institut, Universität Bayreuth, Germany}

tion electrons determine the low-temperature properties of amorphous solids. There is a resonant interaction when acoustic phonons and the tunnelling systems have the same energy, and a relaxation interaction when the sound wave disturbs the equilibrium population of the tunnelling states. The observed acoustic properties of glasses at temperatures between $20 \mathrm{mK}$ and $1 \mathrm{~K}$ (Fig. 1) largely agree with the predictions of the tunnelling model for these two interactions. For example, in a dielectric glass such as amorphous silica (a- $\left.\mathrm{SiO}_{2}\right)$, a maximum in the relative velocity of sound $\Delta v / v$ arises at a temperature $T_{\max }$ when the contribution due to the resonant interaction is just compensated by the contribution of the relaxadecrease with temperature of $v$ above $T_{\max }$ is about 0.5 times the rate of the logarithmic decrease of $v$ below $T_{\max }$ (Fig. 1a). Secondly, the measured $1 / Q$ value corresponding to the plateau in the sound attenuation (Fig. 1b) agrees with the value calculated from the gradients of $v$ above and below $T_{\max }$ using the tunnelling model. Indeed, the presence of a temperature-independent plateau in the attenuation, as well as a maximum and then a logarithmic temperature dependence of the velocity of sound over a wide temperature range, are crucial tests of the basic assumptions of the tunnelling model for the density of states and relaxation times of the tunnelling excitations.

The situation in amorphous metals is not as clear as in dielectric glasses. In amorphous PdSiCu, for example, the tempera-

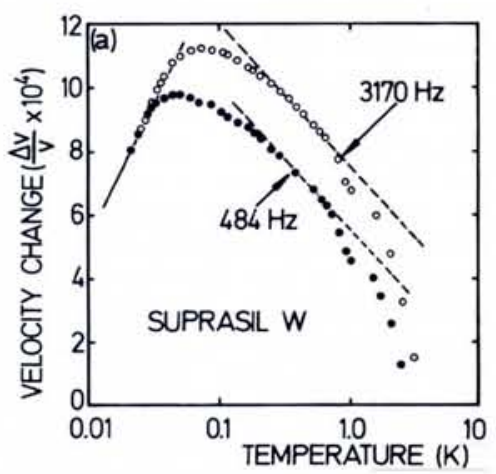
tion interaction. The rate of the logarithmic ture dependence of $v$ below $T_{\max }$ does not depend on the frequency, in perfect agreement with the tunnelling model [2]. However, this remarkable effect has not been observed in other amorphous metals, where disagreement is found between measurements and the predictions of the accepted theory for both acoustic properties [4]. This disagreement is pronounced for a-PdSiCu at very low temperatures [5].

Aside from the general remark that in spite of much work, little is known about the microscopic nature of the tunnelling systems in disordered materials, we are especially interested in understanding lowenergy excitations in these materials, and the interaction of the excitations with phonons and conduction electrons. The critical issue is to establish whether or not the lowfrequency acoustic properties of dielectric and metallic glasses at very low temperatures can still be described by the standard tunnelling model. Other unsolved questions concern the possibility of a low-energy limit for the density of tunnelling states in glasses and the differences between a glass and a disordered polycrystal. For instance, can simple, polycrystalline metals show indications of a glass-like acoustic behaviour such as that found $[2,6]$ in some orientationally or compositionally disordered dielectric crystals, as well as in various alloys.

\section{Ultra-Low Temperature Experiments}

Using the vibrating reed $[2,5]$ and the vibrating wire [5] techniques in combination with nuclear refrigeration, we have extended

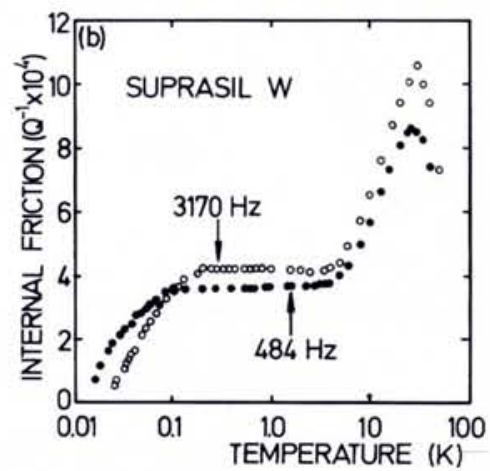

Fig. 1 - Low-temperature acoustic properties of amorphous silica $\left(\mathrm{a}-\mathrm{SiO}_{2}\right.$; Suprasil W) plotted as a function of temperature at two different phonon frequencies (from [2]). a, left) The relative change of the velocity of sound passes through a maximum and shows a logarithmic dependence (indicated by straight lines) on both sides of the maximum. b, right) The sound attenuation (internal friction) exhibits a plateau. 
the low-temperature limit, and thus the energy scale for investigating acoustic properties of solids, by two orders of magnitude (the experiments were at frequencies $\omega / 2 \pi$ between $100 \mathrm{~Hz}$ and $8 \mathrm{kHz}$ with temperatures between $40 \mu \mathrm{K}$ and $1 \mathrm{~K}$ ). The advantages of low-frequency acoustic investigations of glasses have been discussed by Hunklinger and Raychaudhuri [2], and details of samples, experimental methods, and thermalization of the samples during acoustic experiments at ultra-low temperatures, as well as a discussion of non-linear acoustic properties, are covered in several recent publications [5].

Measurements at the ultra-low temperatures were performed in a nuclear refrigeration facility [7] where the sample holders for the vibrating reeds or vibrating wires were screwed to the upper flange of a nuclear refrigeration stage made from copper. Temperatures were determined

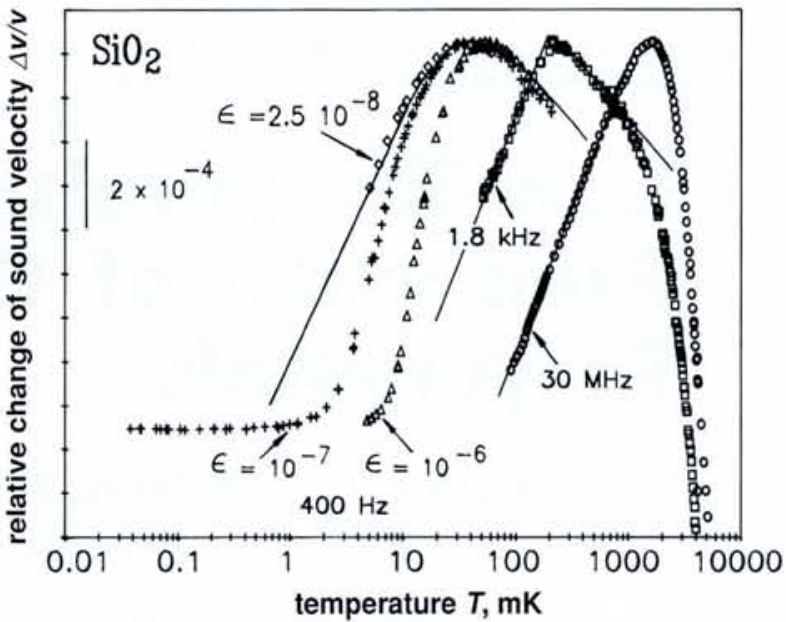

Fig. 2 - Relative change of the velocity of sound in a-SiO (Suprasil-I) at different frequencies. The strains $\varepsilon$ for the data taken at $400 \mathrm{~Hz}$ are calculated from the signals measured at 10 $m K$. The departures from the full line calculated according to the standard tunnelling model for zero strain correspond to non-linear behaviour at very low temperatures. using a platinum NMR thermometer connected to the flange. In order to minimise internal heating, vibration amplitudes at the surface of the reed at the clamped end were typically of the order of $10 \mathrm{~nm}$ corresponding to a maximum strain $\varepsilon$ of $\approx 10^{-7}$; the dissipated energy in the samples is typically some $10^{-15} \mathrm{~W}[5]$. Under these conditions, a- $\mathrm{SiO}_{2}$ shows a reversible, fast thermal response down to $600 \mu \mathrm{K}$ with essentially no difference in temperature compared to the temperature indicated by the thermometer. However, below approximately $300 \mu \mathrm{K}$ the temperature of the sample is not known anymore.

For samples of polycrystalline metals such as $\mathrm{Ag}$ and $\mathrm{Cu}$, thermal hysteresis was not observed even at $40 \mu \mathrm{K}$. Owing to the higher resonance frequencies for the vibrating wire experiments compared to the vibrating reed measurements, and to the low thermal conductivities of thin, supercon-

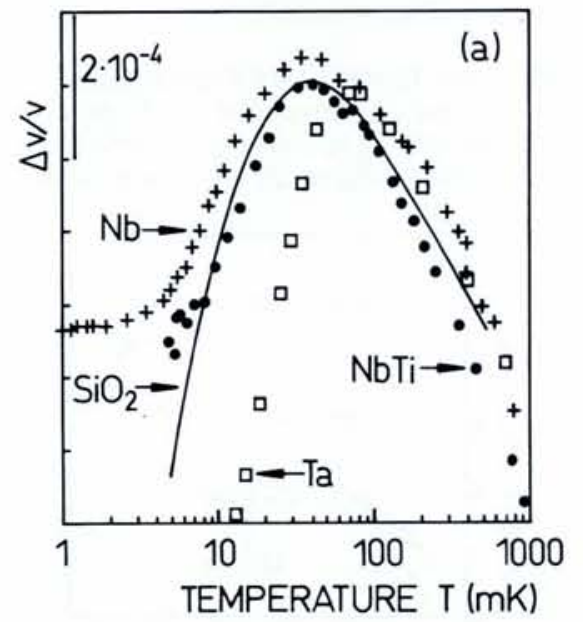

Fig. 3 - The relative change of the velocity of sound in a-SiO $\mathrm{S}_{2}$ and the crystalline superconductors $\mathrm{Nb}, \mathrm{NbTi}$ and $\mathrm{Ta}$ at $0.40 \mathrm{kHz}, 1.5$ $\mathrm{kHz}, 0.37 \mathrm{kHz}$, and $5.5 \mathrm{kHz}$, respectively, demonstrates the similarity between these materials. The $\Delta v / v$ scale for $\mathrm{Nb}(\mathrm{Ta}$ ) has to be divided by a factor 4 (10). ducting wires (diameters typically 20-150 $\mu \mathrm{m})$, internal heating due to dissipation limited measurements on $\mathrm{Al}, \mathrm{Ta}, \mathrm{Nb}$, and $\mathrm{NbTi}$ wires to above several $\mathrm{mK}$.

\section{Testing the Tunnelling Model}

Fig. 2 shows $\Delta v / v$ as a function of temperature for $\mathrm{a}-\mathrm{SiO}_{2}$ at three different frequencies. The overall behaviour observed above $20 \mathrm{mK}$ is typical of amorphous dielectrics and is consistent with predictions of the standard tunnelling model. However, below $10 \mathrm{mK}$ and at $400 \mathrm{~Hz}$ we observe straindependent deviations from the logarithmic decrease of $v$. Moreover, there are two other experimental results which disagree with expectations, namely a shift of $T_{\max }$ with strain and saturation of $v$ at low temperatures. One should note that the dependence of the velocity of sound on the acoustic intensity (or strain) is opposite to that which would be expected if heating of the sample was responsible for the effect.

The strain-dependent behaviour of the velocity of sound is observed when the energy of the sound waves is of the order of the thermal energy $k_{\mathrm{B}} T$, where $k_{\mathrm{B}}$ is Boltzmann's constant. We have been able to describe the observed "anomalies" of $v$ by introducing a change in the population number of the tunnelling states created by the waves. The non-linear behaviour of the velocity of sound results from a dependence of the population number of the energy states of the tunnelling systems on the strain. Consistent with this explanation is the observation that the dependence, in the temperature range of the measurements, of the sound attenuation on the strain is much smaller because the attenuation only depends on the relaxation interaction between phonons and thermal scattering in our frequency range; this was indeed observed. However, the attenuation $1 / Q$ followed closely a linear dependence on $T$ at temperatures below those for the plateau, in disagreement with the prediction $1 / Q \sim T^{3}$ of the tunnelling model.
An important theoretical result of Parshin [8], namely a logarithmic dependence of $\Delta v / v$ on the time of the experiment, has also been confirmed by us more recently.

\section{Glassy Dielectrics versus Crystalline Superconductors}

Measurements [4] at temperatures well below the critical temperature for superconductivity have shown that the acoustic properties of amorphous superconductors resemble those of amorphous dielectrics because the number of normally conducting electrons is negligible in both cases. We wanted to investigate whether the acoustic properties of superconducting crystalline materials could be compared with those of amorphous dielectrics.

Fig. 3 shows $\Delta v / v$ as a function of temperature for three different polycrystalline superconductors (Nb, $\mathrm{NbTi}, \mathrm{Ta}$ ) along with the data of Fig. 2 for a- $\mathrm{SiO}_{2}$. The variations of $\Delta v / v$ as well as those of the sound attenuation [5] match those of the amorphous dielectrics; even the strain dependence of $\Delta v / v$ of the crystals is the same as for a- $\mathrm{SiO}_{2}$ as long as self-heating effects are negligible. The saturation of $v$ below $5 \mathrm{mK}$ observed for $\mathrm{Nb}$ could be related to the phenomenon which saturates $v$ in a-SiO ${ }_{2}(\mathrm{Fig}$. 2). We exclude sample heating effects since data for $\mathrm{Nb}$ wires in a $\mathrm{Cu}$ matrix, which greatly enlarges the thermal conductivity of the sample, show the same behaviour.

Applying the tunnelling model we obtain, from the logarithmic slopes of the velocity of sound and from the plateau of the attenuation, values for the tunnelling density of states of $\mathrm{Nb}, \mathrm{NbTi}$ and $\mathrm{Ta}$ which are comparable to those found for $\mathrm{a}-\mathrm{SiO}_{2}$ and a-PdSiCu [5].

Other clear-cut experimental evidence which speaks for a similar energy dependence of relaxation times of tunnelling systems in polycrystals is the frequency dependence of the onset of the plateau in $1 / Q$. According to the standard tunnelling model, this onset should follow a $\omega^{1 / 3}$ dependence, as has been observed by us in $\mathrm{NbTi}$.

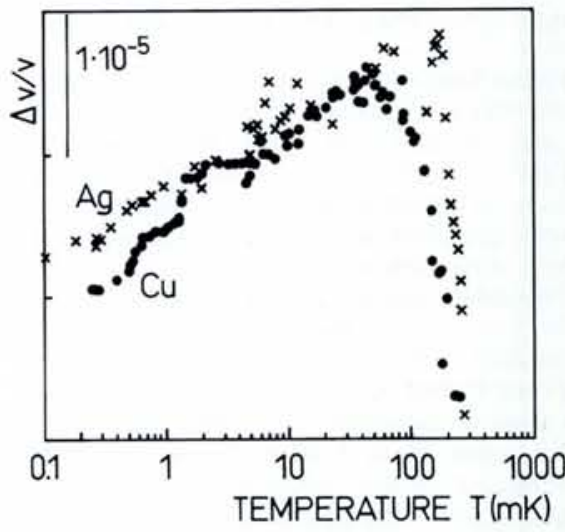

Fig. 4 - Relative change of the velocity of sound measured using a $\mathrm{Ag}$ vibrating reed and a $\mathrm{Cu}$ vibrating wire at $250 \mathrm{~Hz}$ and 300 $\mathrm{Hz}$, respectively, showing the glass-like behaviour of these polycrystalline metals. 


\section{Normal Polycrystalline Metals}

The similarity in the acoustic behaviour of polycrystalline superconductors and amorphous dielectrics prompted us to look for indications of glass-like behaviour in simple, normally conducting metals. Fig. 4 shows $\Delta v / v$ as a function of temperature for polycrystalline $\mathrm{Ag}$ and $\mathrm{Cu}$. The low-frequency, low-temperature acoustic properties are similar to those of a-SiO ${ }_{2}$ and a-PdSiCu: for the attenuation, there is a plateau between $60 \mathrm{mK}$ and $600 \mathrm{mK}$ followed by a decrease proportional to $T^{1 / 3}$; for the velocity of sound, there is a maximum at $100 \mathrm{mK}$ and then a logarithmic decrease. The velocity follows a logarithmic temperature dependence over three decades in $T$ [5], the best "glassy behaviour" of $v$ ever observed. A decrease of the impurity concentration in $\mathrm{Ag}$ from $100 \mathrm{ppm}$ to $1 \mathrm{ppm}$ had only a small influence on the results. The anomalies decreased under heat treatment, and could be recovered by cold-rolling, so we relate the observed acoustic properties mainly to lattice defects rather than to impurities.

The observed properties of $\mathrm{Ag}$ and $\mathrm{Cu}$ are signatures of low-energy excitations with a wide distribution of energy and relaxation times. The temperature dependence of the velocity of sound in the two metals is about 100 smaller than the dependence in $\mathrm{a}-\mathrm{SiO}_{2}$, and about 25 times smaller than in aPdSiCu. The density of states of the lowenergy excitations in polycrystalline $\mathrm{Ag}$ and $\mathrm{Cu}$ would thus be roughly $1 \%$ of the density in $\mathrm{a}-\mathrm{SiO}_{2}$ or a-PdSiCu if the deformation potentials in the disordered state are comparable, and if the standard tunnelling model can be applied. However, in view of the recent theory incorporating non-linear phonon interaction [8] and the complexity of electron interaction in tunnelling systems [9], the temperature dependence of $v$ can hardly be used to reliably estimate the density of states of low-energy excitations in metals, where the number of conducting electrons is very large. Moreover, in contrast to a-SiO 2 , a-PdSiCu and polycrystalline superconductors, we find no saturation and no strain dependence of the velocity of sound in $\mathrm{Ag}$ and $\mathrm{Cu}$ (for $10^{-4}>\varepsilon \geq 10^{-6}$ ) and of the internal friction of $\mathrm{Ag}$ to the lowest temperatures attained so far (an observed dependence of $1 / Q$ on strain for $\mathrm{Cu}$ may result from dislocation damping and not from possible glassy behaviour). The observation of glass-like acoustic behaviour in polycrystalline metals has recently been extended to $\mathrm{Pt}$.

\section{Conclusions}

The similarity between the acoustic properties of monoatomic polycrystalline metals $(\mathrm{Ag}, \mathrm{Cu}, \mathrm{Pt})$, superconductors ( $\mathrm{Al}, \mathrm{Ta}, \mathrm{Nb}$, $\mathrm{NbTi}$, and glasses is undoubtedly more significant than the deviations at finite strain and very low temperatures from the predictions of the standard tunnelling model. It may be not so surprising to find tunnelling systems or low-energy excitations of some kind in a polycrystalline material. However, excitations associated with defects or impurities in polycrystals are expected to have discrete energies, very different from the energy-independent excitation spectrum of glasses. One possibility is that strain interactions between localized atomic defects in a crystalline lattice lead to a broad distribution of energies and relaxation times [10].

However, the perfectly logarithmic increase of the velocity of sound over at least three orders of magnitude in temperature for $\mathrm{Ag}$ and $\mathrm{Cu}$, which has no parallel in previous measurements on glasses, and the close similarity of the temperature and strain dependence of the acoustic properties of the polycrystalline superconductors to those of the dielectric a- $\mathrm{SiO}_{2}$, provide clear support for the existence of a wide distribution of energies and relaxation times. The results thus indicate that glass-like anomalies are far more widely observed than expected they would seem to question our present understanding of disorder. It remains to be seen if the differences between the lowfrequency acoustic properties of a glass (with random disorder) and a polycrystal (with distinct defects) is qualitative instead of merely quantitative, in which case a new theoretical approach rather than an extension of the standard tunnelling model would be needed to explain the observed properties at very low temperatures.

[1] "Amorphous Solids: Low Temperature Properties", in Topics in Current Physics 24, Ed. W.A. Phillips (Springer, 1981).

[2] Hunklinger S. \& Raychaudhuri A.K, in Prog. in Low Temp. Physics IX (NorthHolland, 1986) Ed.: D.F. Brewer; idem, Z. Phys. B - Cond. Matter 57 (1984) 113.

[3] Phillips W.A., J. Low Temp. Phys. 7 (1972) 351; Anderson P.W., Halperin B.I. \& Varma C.M., Phil. Mag. 25 (1972) 1.

[4] Esquinazi P. et al., Z. Phys. B 64 (1986) 81; idem, Phys. Rev. B 37 (1988) 7819.

[5] Esquinazi P., König R. \& Pobell F., Z Phys. B 87 (1992) 305; König R., Esquinazi P. \& Pobell F., J. Low Temp. Phys. 90 (1993) 55. [6] Hikata H. \& Elbaum C., Phys. Rev. Lett. 54 (1985) 2418; Birge N., et al., Phys. Rev. B 36 (1987) 7685; Raychaudhuri A.K. \& Pohl R.O., Phys. Rev. B 46 (1992); Van Cleve J.E., Pohl R.O. \& Raychaudhuri A.K., Phonon Scattering in Cond. Matter VII, Proc. 7th Int. Conf. on Phonon Scattering in Cond. Matter, Cornell, USA, Eds.: M. Meissner \& R.O. Pohl (Springer, 1993); Ackerman D.A., et al., Phys. Rev. B 23 (1981) 3886.

[7] Gloos K., et al., J. Low Temp. Phys. 73 (1988) 101.

[8] Parshin D.A., Z. Phys. B - Cond. Matter (to be published).

[9] Kagan Yu. \& Prokof'ev N.V., Sov. Phys. JETP 70 (1990) 957.

[10] Klein M., et al., Phys. Rev. B 18 (1978) 5887.

\section{Professor of Molecular and Laser Physics mlf}

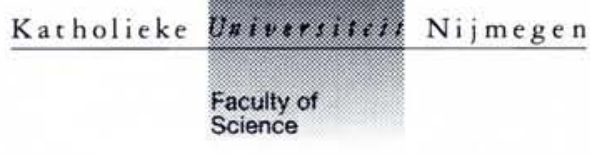

The Department of Physics at the University of Nijmegen, The Netherlands, invites applicants for the position of professor of experimental physics.

Candidates should have proven record of success in carrying out original research in the field of molecular and laser physics. The chosen candidate is expected to develop a new research program that enhances ongoing research in the group on molecular spectroscopy, collision dynamics and the development and application of advanced laser techniques. Participation in applied research is also expected. Present applied projects include trace gas detection and analysis of combustion processes.
Teaching undergraduate and graduate physics courses is an important aspect of this position.

Foreign candidates must be able to teach in Dutch within two years.

For further information please contact Prof J. Kuijpers, Chairman of Search Committee, tel. +3180652080 or Prof D.H. Parker, tel. +31 80653423 .

Applicants are requested to send a C.V. and list of publications by September 13, 1993 to University of Nijmegen, Faculty of Science, Personnel Office, Toernooiveld 1, 6525 ED Nijmegen, The Netherlands under reference 24-93. 